\title{
Effect of Cross-linking Reactions on Evolution Mechanism of Crystalline-Amorphous Carbon during Bituminous Coal Carbonization
}

\author{
Dongdong Liü,a, Jihui $\mathrm{GaO}^{2, \mathrm{~b}}$, Shaohua $\mathrm{Wu}^{3, \mathrm{c}}$ and Yunkun Qin ${ }^{4, \mathrm{~d}}$ \\ ${ }^{1-4}$ School of Energy Science and Engineering, Harbin Institute of Technology, 92, West Dazhi Street, \\ Harbin 150001, P. R. China \\ a liudongdong84@sina.comb gaojh@hit.edu.cncwush@hit.edu.cn ${ }^{\text {d }}$ qinyk@hit.edu.cn
}

Keywords: crystalline-amorphous carbon, cross-linking reactions, bituminous coal carbonization

\begin{abstract}
Crystalline carbon and amorphous carbon are two of the major carbon structures found in coal. The influence of cross-linking reactions (CLRs) on the evolution mechanism of crystalline-amorphous carbon during bituminous coal carbonization was studied using X-ray and Raman crystallographic methods. These results showed that firstly low-temperature CLRs enhanced the transformation of amorphous carbon into crystalline carbon at the begin of carbonization, then affected the mean size of crystalline structures, and finally facilitated degradation processes that resulted in the transformation of crystalline carbon into amorphous carbon. However, different effects were observed for moderate-temperature CLRs on crystalline-amorphous carbon. Firstly, the disruption of weak bonds led to the transformation of crystalline carbon into amorphous carbon. Then moderate-temperature CLRs led to the appearance of small layers in amorphous carbon structures and initiated the transformation of amorphous carbon into crystalline carbon. Finally, the amount of crystalline carbon with respect to amorphous carbon continued to grow.
\end{abstract}

\section{Introduction}

Coal-based activated carbon has been employed as an adsorbent material for the removal of air pollutants [1]. The method of physical preparing coal-based activated carbon is classified into two parts [2]: carbonization and activation. The structural characteristics of char have important effects on the activation process and the resulting activated carbon. Meijun Wang et al. [3] have suggested that when carbon conversion is below $10 \%$, some smaller structures (amorphous carbon structures) are preferentially removed during the gasification process of chars. T. Wigmans et al. [4] have suggested that activating gases first remove amorphous carbon to initiate the development of a pore structure, after which active sites on crystalline carbon structures react with activating gases to form the final pore structure and chemical surface properties of the char. Carbonization is a complicated and multifaceted process during which the macromolecular structure of coal, including its amorphous-crystalline carbon structures, undergoes chemical-thermal destruction and depolymerization reactions that form char [5]. Therefore, the carbonization process associated with the structural modification of char is regarded as an important part of the preparation of coal-based activated carbon.

According to the literature [6], crystalline carbon and amorphous carbon are the two major carbon structures in coal. Crystalline carbon, which is a structure between graphitic and amorphous structures, is also called graphite-like or turbostratic carbon. Crystalline carbon, different from large graphitic structures, has a very small structure. In addition to crystalline carbon, amorphous carbon, which has a highly disordered structure, is an important component of coal. The macromolecular structure of coal is composed primarily of these crystallites, which are connected by side chains and intermingled with amorphous carbon structures.

Zhiqing Wang et al. [7] have found that some low-molecular fractions (THFS) transform into intermediate molecular fractions (THFI) by way of cross-linking reactions (CLRs) and that these THFI fractions are capable of cross-linking with each other to develop solid products during coal liquefaction. Thus, the structural development and transformation of crystalline carbon and amorphous carbon may be influenced by cross-linking reactions. Cross-linking reactions can be 
divided into low- and moderate-temperature reactions [8]. Some oxygen-containing groups are broken at low-temperatures and cross-linked to form $\mathrm{CO}_{2}$ and $\mathrm{H}_{2} \mathrm{O}$, while moderate-temperature cross-linking is related to the decomposition of methyl groups by displacement reactions to form $\mathrm{CH}_{4}$. It remains unclear whether a relationship exists between the structural development and transformation of crystalline and amorphous carbon structures and cross-linking reactions during carbonization.

The objective of this study was to examine how the evolution process of crystalline-amorphous carbon is altered by CLRs during carbonization. These parameters were studied using X-ray and Raman crystallographic methods. This work can be used to directionally control the structure of char, which allows for improvements in the quality of coal-based activated carbon.

\section{Experimental procedure}

\subsection{Materials}

Datong bituminous coal from Western China was used in this study. Data from proximate and ultimate analyses of raw coal samples are given in Table 1 . The coal was pulverized to particles 250-380 $\mu \mathrm{m}$ in size and washed with deionized water. The washed coal samples were dried at $150{ }^{\circ} \mathrm{C}$ in nitrogen for $20 \mathrm{~h}$ to remove the water and then stored in a glass bottle prior to carbonization studies.

Table 1. Proximate and ultimate analyses of experimental coal sample

\begin{tabular}{clcl}
\hline \multicolumn{2}{c}{ Proximate analysis (wt \%) } & \multicolumn{2}{c}{ Ultimate analysis (wt \%) } \\
\hline$M_{\mathrm{ad}}$ & 1.10 & $\mathrm{C}_{\mathrm{daf}}$ & 72.09 \\
$V_{\mathrm{daf}}$ & 32.63 & $\mathrm{H}_{\mathrm{daf}}$ & 4.63 \\
$A_{\mathrm{d}}$ & 5.75 & $\mathrm{O}_{\mathrm{daf}}{ }^{a}$ & 14.61 \\
$\mathrm{FC}_{\mathrm{ad}}$ & 60.52 & $\mathrm{~N}_{\mathrm{daf}}$ & 1.61 \\
& & $\mathrm{~S}_{\mathrm{daf}}$ & 0.21 \\
\hline
\end{tabular}

${ }^{a}$ By differenc

\subsection{Preparation of acid-treated coal samples}

In accordance with standard methods [9], the demineralization of the coal samples was performed as follows: $100 \mathrm{ml}$ of $5 \mathrm{~N} \mathrm{HCl}$ was added to $15 \mathrm{~g}$ of coal samples and stirred for $12 \mathrm{~h}$ at $40{ }^{\circ} \mathrm{C}$ under a nitrogenous atmosphere. Then, the mixture was filtered and mixed with $120 \mathrm{ml}$ of $30 \% \mathrm{HF}$ and stirred again at for $12 \mathrm{~h}$ at $40{ }^{\circ} \mathrm{C}$ under a nitrogenous atmosphere. After filtration, the mixture was washed with distilled water to remove chloride ions. Finally, the HCl/HF-washed coal samples were dried at $100{ }^{\circ} \mathrm{C}$ in nitrogen for $20 \mathrm{~h}$. After $\mathrm{HCl} / \mathrm{HF}$-treatment, the content of ash in the treated coal samples is below $1 \mathrm{wt} . \%$. These samples are denoted " $\mathrm{S}$ " ( $\mathrm{HCl} / \mathrm{HF}$ treatment).

\subsection{Preparation of oxidized coal samples}

After the demineralized procedure, some samples were oxidized in open containers at $200{ }^{\circ} \mathrm{C}$ for $15 \mathrm{~h}$. These samples are denoted "SO15 h".

\subsection{Carbonization}

The carbonization experiments were performed on coal samples in a three-stage fixed-bed reactor at a fixed heating rate of $8{ }^{\circ} \mathrm{C} / \mathrm{min}$ from room temperature to a final temperature of $700{ }^{\circ} \mathrm{C}$ under $\mathrm{N}_{2}$ atmosphere at flow rate of $600 \mathrm{ml} / \mathrm{min}$, and samples were held at the final temperature for $30 \mathrm{~min}$. Finally, the coal was quenched to room temperature under a $\mathrm{N}_{2}$ atmosphere.

\subsection{Measurement analysis}

2.5.1 FTIR: Thesamples were analyzed by FTIR in the $400-4000 \mathrm{~cm}^{-1}$ range employing standard pellets of $\mathrm{KBr}$ matrix, and 200 scans were performed at a resolution of $4 \mathrm{~cm}^{-1}$.

2.5.2 TG: A TG analyzer was used to measure the carbonization process of the samples under a stream of high purity $\mathrm{N}_{2}$ at a flow rate of $60 \mathrm{ml} / \mathrm{min}$. The carbonization conditions used are given above. 
2.5.3 X-ray: After carbonization, the char powder samples were investigated using a $\mathrm{D} / \mathrm{max}$-rb $\mathrm{X}$-ray diffractometer. The samples were measured in the $2 \theta$ range from $5^{\circ}$ to $85^{\circ}$ using a scan rate of $3 \%$ min to obtain structural information regarding the char crystallinity of the samples. In the XRD spectra, the (002) peak and the (10) peak were used to analyze $L_{\mathrm{c}}$ and $L_{\mathrm{a}}$, respectively. The data of $d_{002}$ were obtained using Bragg's equation.

2.5.4 Raman: The chars were examined with Raman Spectroscopy using a $532 \mathrm{~nm}$ wavelength. The Raman spectra data were carefully acquired in the range of 500-1800 $\mathrm{cm}^{-1}$, including first-order bands. The instrument spectra resolution used was less than $2 \mathrm{~cm}^{-1}$, and spectra were obtained at 60 $\mathrm{s}$ intervals. During measurement, no effects from the inorganic matter were detected, which is in accordance with the findings of Green et al. [10] and Bar-ziv et al [11].

2.5.5 Solvent swelling experiments: To analyze the variation in cross-linking within the samples, Liotta's solvent swelling method was used [12]. The swelling behavior inside the macromolecular network of the samples was measured as follows: $200 \mathrm{mg}$ of coal and char samples were centrifuged in a tube for $10 \mathrm{~min}$ at $4000 \mathrm{rev} / \mathrm{min}$ to determine the height of the sample, $h_{1}$. The samples were then mixed with excess pyridine, and after $24 \mathrm{~h}$, the samples were centrifuged to measure the height of the sample again, $h_{2}$. The ratio of the volume of the samples was calculated as $Q_{\mathrm{v}}=h_{2} / h_{1}$. Using Solomon's methods [8], the $Q_{\mathrm{v}}$ values were standardized between 0 and 1 with an $X$ parameter. $1-X=1$ was used for the coal, and $1-X=0$ was used for the maximally cross-linked residue.

$$
X=\frac{Q_{v, \text { coal }}-Q_{v, \text { residue }}}{Q_{v, \text { coal }}-1}
$$

\section{Results and discussion}

\subsection{FTIR analysis of coal}

The FTIR spectra of the two samples at room temperature are given in Fig.1. The aliphatic C-H region is between $2830 \mathrm{~cm}^{-1}$ and $2953 \mathrm{~cm}^{-1}$, which indicates the vibration adsorption of asymmetric $\mathrm{CH}_{2},-\mathrm{CH}_{3}$ stretching and symmetric $\mathrm{CH}_{2}$ stretching. The carboxyl groups were obtained at 1700 $\mathrm{cm}^{-1}$. The band near $1460 \mathrm{~cm}^{-1}$ is assigned to the alkyl chain structure $\mathrm{CH}_{2}$ and $\mathrm{CH}_{3}$ deformation vibrations [13].

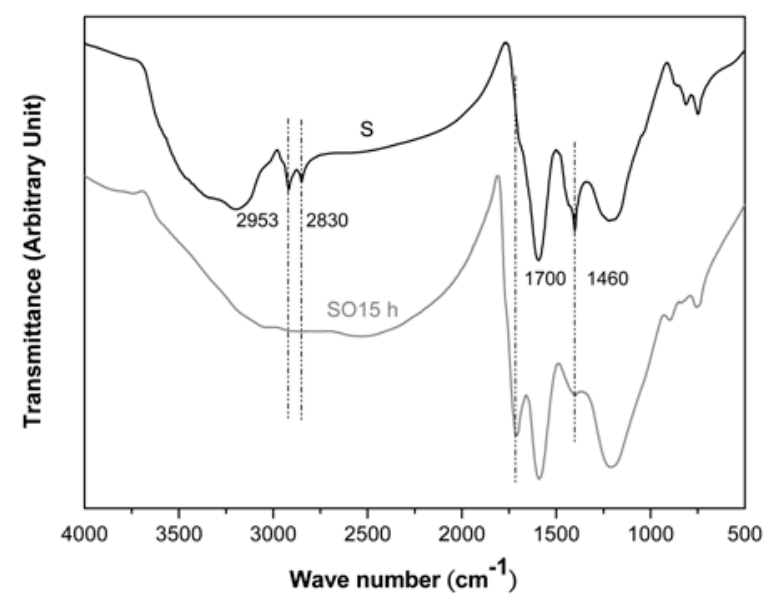

Figure 1 FTIR spectrum of chars from S and SO15 h samples at room temperature

From Fig. 1, the aliphatic C-H is clearly observed in the S sample, and disappears in the SO15 h sample due to the pre-oxidation treatment. The alkyl chain structures $\mathrm{CH}_{2}$ and $\mathrm{CH}_{3}$ show the same tendency. After the oxidation treatment, the typical carboxyl groups are found in the SO15 h sample [5]. 


\subsection{Carbonization process of coal}

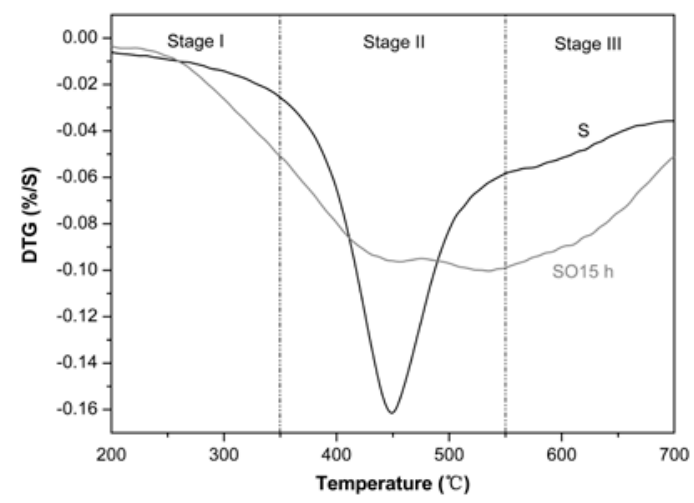

Figure 2 DTG curves of S and SO15 h samples carbonization

Fig. 2 shows the DTG curves of two samples of carbonized coal. The carbonization stage is indicated by moisture and volatile lumps. The carbonization behaviors of the two samples, $\mathrm{S}$ and $\mathrm{SO} 15 \mathrm{~h}$, are different during these three stages. In Stage I, the rates of weight loss and thermal decomposition of the two samples decrease in the following order: SO15 h > S. The changes observed in the S sample include the breakage of weak bonds between basic structural units and a gradual increase in the concentration of free radicals. Because more oxygen functional groups (such as carboxyl) are created during pre-oxidation and are more easily broken to generate oxygen-containing gases during stage I, the rate of weight loss of SO15 h is greater than that of S, and abundant free radicals are produced by SO15 h.

In stage II, bituminous coal can melt during this plastic stage, the rates of weight loss and thermal decomposition of the two samples differ. Tar and some hydrocarbons are the primary products of devolatilization during this stage and are related to the macromolecular structure of the samples. Some aliphatic $\mathrm{CH}$ groups available for hydrogen transfer play an important role in the development of plasticity [15], during which the available hydrogen saturates the free radicals produced by the carbonization process to generate mesophase and anisotropic coke [16]. However, when free radical species cannot be saturated by hydrogen transfer, the isotropic coke structure and cross-linking will more easily form from the union of free radicals. In the process of plasticity, microcrystalline structures are rearranged to form more ordered, less porous, and less reactive structures. At approximately $450{ }^{\circ} \mathrm{C}$, a sharp peak in the rate of weight loss is detected for the $\mathrm{S}$ sample, but stage II is scarcely extant for $\mathrm{SO} 15 \mathrm{~h}$ sample at this temperature. Only two carbonization stages (stages I and III) are observed for the SO15 h samples. For the S samples, a sharp peak in the rate of thermal decomposition indicates that more aliphatic $\mathrm{CH}$ groups in aliphatic chain structures are broken to provide available hydrogens to saturate the free radicals. Upon heating, the S sample undergoes a plastic stage. However, the SO15 h samples pass directly into stage III, owing to the disappearance of their aliphatic CH groups after pre-oxidation, which results in serious damage to the thermoplastic properties of these samples.

Change in volatile release and the decomposition of oxygenated groups and ring condensation continue during stage III. More volatiles are released from the SO15 h samples than the S samples, and the decreasing char content results in different char structures for these samples.

\subsection{Analysis of cross-linking reactions}

From Fig. 3, it can be observed that the curves of the two samples differ. When the carbonization temperature is less than $300{ }^{\circ} \mathrm{C}$, the $1-\mathrm{X}$ index of the $\mathrm{S}$ sample is 1.05 , which shows a decrease in cross-linking. This phenomenon may occur because more covalent bonds are cleaved than are generated. The SO15 h samples show a sharp reduction in their 1-X index in this temperature region. Previous studies [8] have indicated that more oxygenated functional groups (such as carboxyl) are broken at low temperatures to produce $\mathrm{CO}_{2}$, which may play an important role in increasing the frequency of low-temperature cross-linking processes within the coal macromolecule. 


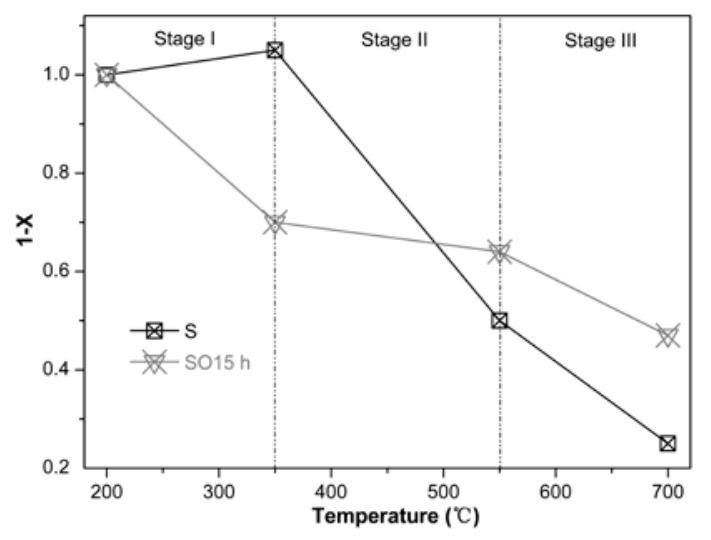

Figure 3 Comparison of solvent swelling ratios for coals of S and SO15 h samples during carbonization

When the carbonization temperature is between 300 to $500^{\circ} \mathrm{C}$, the curve of the S sample sharply declines. The moderate-temperature cross-linking observed results from the decomposition of methyl groups by displacement reactions to form $\mathrm{CH}_{4}$ [8]. However, the plastic state is limited to moderate-temperature cross-linking reactions. As moderate-temperature cross-linking density increases, the fluidity of coal will decrease, resulting in a poorly ordered structure. However, the extent of moderate-temperature cross-linking in SO15 h samples lacking methyl groups due to pre-oxidation is low.

The different trends in cross-linkage density of the two samples are similar during condensation. By adjusting their orientations and distances, these aromatic nuclei can combine to develop larger layers. This results in an increase in the density of cross-links.

\subsection{Raman and X-ray analysis of chars}

Because organic coal is a complex structure, its structure cannot be fully solved using one method [17]. The crystalline-amorphous carbon structure observed during carbonization was further studied with the help of X-ray and Raman crystallography.Some structural parameters of chars can be also obtained by means of Raman spectroscopy [18]. The assignment of the 10 bands in the Raman spectroscopy of chars is summarized [9]. The four main bands or groups $\left(G, G_{R}+V_{L}+V_{R}, D\right.$ and S) account for a large proportion of the Raman peak area in two samples, as a result, only these bands or groups will be discussed in this work. The content of crystalline carbon $\left(C_{\mathrm{cr}}\right)$ in coal can be found from the intensity of the $\mathrm{D}$ and $\mathrm{G}$ compared to the total intensity ( $\left.I_{\text {Total }}\right)$ [19]. The $\mathrm{G}_{\mathrm{R}}+\mathrm{V}_{\mathrm{L}}+\mathrm{V}_{\mathrm{R}}$ bands can be described as the rather small aromatic structures (3-5 rings). The $\mathrm{S}$ band mainly stands for the sp3-rich structures in which the $C_{\text {alkyl }}-C_{\text {aryl }}$ bonds and methyl carbon linking to an small aromatic ring are formed, and can be also regarded as a main measure of cross-linking density. The $S$ and $G_{R}+V_{L}+V_{R}$ bands are very useful for the study of representative structures of amorphous carbon. The structural parameters of the two samples during carbonization as obtained by X-ray and Raman crystallography are shown in Fig. 4 and Fig. 5. 


\subsubsection{S sample}

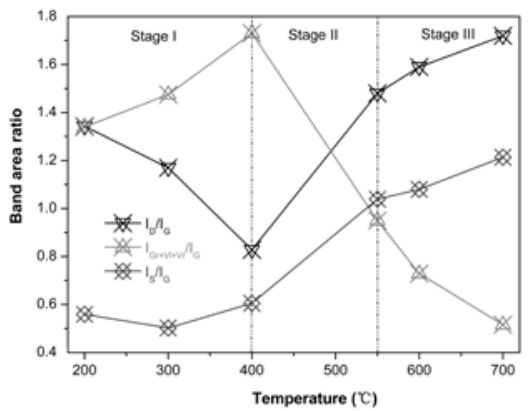

(a)

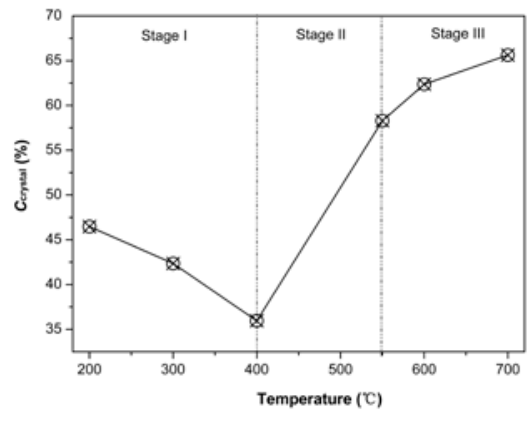

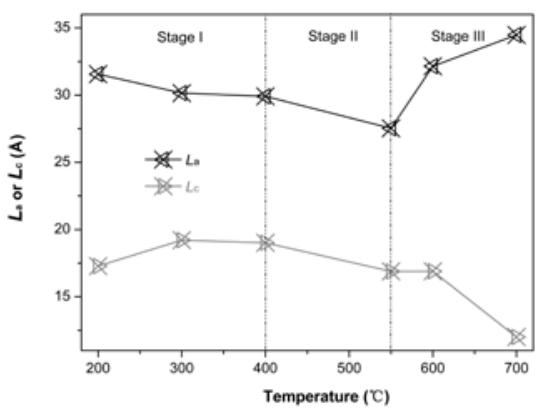

(b)

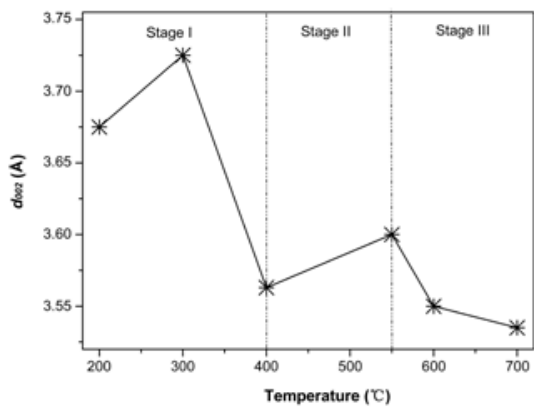

(d)

Figure $4 \mathrm{X}$-ray and Raman parameters of $\mathrm{S}$ sample during carbonization
(a) $I_{\mathrm{D}} / I_{\mathrm{G}}, I\left(\mathrm{Gr}+\mathrm{Vl}+\mathrm{Vr}_{\mathrm{r}}\right) / I_{\mathrm{G}}$ and $I_{\mathrm{S}} / I_{\mathrm{G}}$; (b)
(b) $L_{\mathrm{a}}$ and $L_{\mathrm{c}}$,
(c) $C_{\text {cryst }}$;
(d) $d_{002}$

Curves representing the relationships between the structural parameters and the heating temperature of S samples are listed in Fig. 4. In the thermal destruction phase, the $C_{\mathrm{cr}}$ parameter exhibits a slight decrease, the $L_{\mathrm{a}}$ parameters also decrease and the $d_{002}$ and $L_{\mathrm{c}}$ parameter increases. The changes in the $C_{\mathrm{cr}}$ and $L_{\mathrm{a}}$ parameters can be explained by the decomposition process in crystallites resulting from the dissolution of weak bonds between the basic crystallite structure units, while the decrease in $I_{\mathrm{D}} / I_{\mathrm{G}}, I_{\mathrm{S}} / I_{\mathrm{G}}$ and the increase in $I_{(\mathrm{GR}+\mathrm{VL}+\mathrm{VR})} / I_{\mathrm{G}}$ can be observed in Fig. 4 . These changes indicate that the crystalline carbon structure has started to depolymerize and convert into an amorphous carbon structure. In other words, a decrease in cross-linking density is responsible for a transformation from large aromatic rings to the rather small ones. An increase in the $L_{\mathrm{c}}$ parameter without a corresponding increase in the $C_{\mathrm{cr}}$ parameter is illustrated by the mechanism, namely, the uniting of layers is the result of their changed orientation rather than their growth. The union of layers is accompanied by the development of an imperfect structure and greater spacing $d_{002}$.

The $C_{\mathrm{cr}}$ parameter begins to increase during the moderate-temperature cross-linking stage. There are a great increase in $I_{\mathrm{D}} / I_{\mathrm{G}}$ and decrease in $I_{(\mathrm{GR}+\mathrm{VL}+\mathrm{VR})} / I_{\mathrm{G}}$ at temperature between 400 and $550^{\circ} \mathrm{C}$, indicating that cyclic polymerization processes and transformation of some small aromatic rings into large ones resulting from moderate-temperature CLRs (the more formation of $C_{\text {alkyl }}-C_{\text {aryl }}$ bonds) can result in an increase in the relative proportion of the ordered phase. The decreases in $L_{\mathrm{a}}$ and $L_{\mathrm{c}}$ may be a consequence of the appearance of small layers in the amorphous carbon structure that come together without coalescence due to their disadvantageous positions or adverse orientations and the formation of cross-links in relation to larger neighbors as parameter $d_{002}$ reaches its maximum value. These findings can be attributed to the appearance of new crystallite nuclei, which produce larger interlayer distances.

The number of new crystallite nuclei continues to increase as the carbonization temperature rises. The new and old crystallite nuclei rearrange their orientations to coalesce and develop larger layers due to their highly reactive state, while the formation of new $C_{\text {alkyl }}-C_{\text {aryl }}$ bonds are further accelerated. These changes lead to an increase in La and the transformation of these small aromatic rings into large ones. The number of crystalline carbon structures increases due to the prevalence of new crystallites with smaller $L_{\mathrm{c}}$ parameters, which results in a decrease in the mean value of $L_{\mathrm{c}}$. However, the effect of the presumed stretching of crystalline layers is due to a decrease in the number of layers. 


\subsubsection{SO15 h sample}

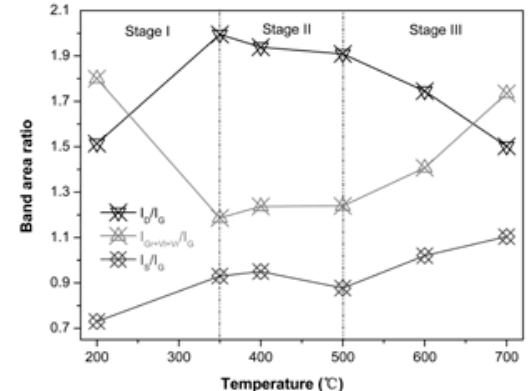

(a)

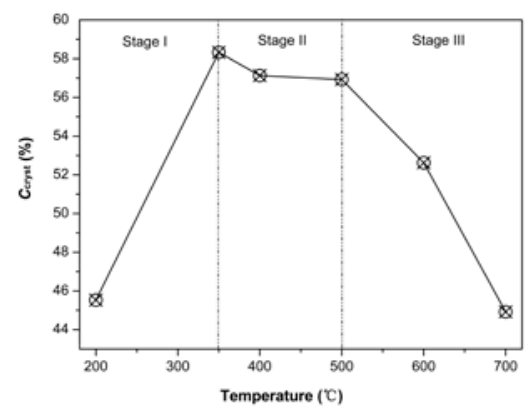

(c)

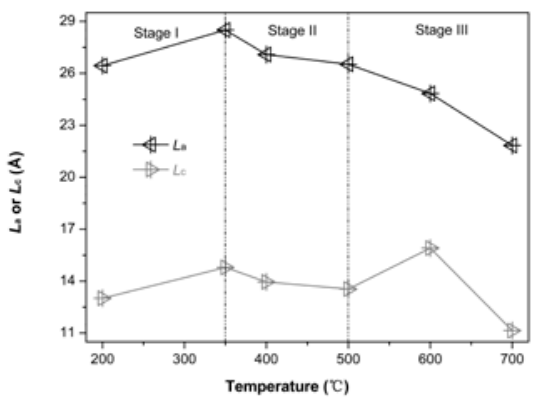

(b)

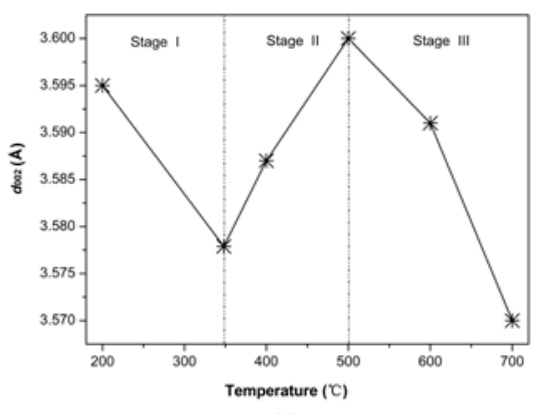

(d)

Figure $5 \mathrm{X}$-ray and Raman parameters of SO15 h sample during carbonization

(a) $I_{\mathrm{D}} / I_{\mathrm{G}}, I\left(\mathrm{Gr}+\mathrm{Vl}+\mathrm{Vr}_{\mathrm{r}}\right) / I_{\mathrm{G}}$ and $I_{\mathrm{S}} / I_{\mathrm{G}}$; (b) $L_{\mathrm{a}}$ and $L_{\mathrm{c}}$, (c) $C_{\text {cryst }}$; (d) $d_{002}$

The relationships of the structural parameters and the heating temperature of SO15 h samples are shown in Fig. 5 . In the stage I, the $C_{\mathrm{cr}}, L_{\mathrm{c}}$ and $L_{\mathrm{a}}$ parameters increased, while the $d_{002}$ parameter decreased. The $C_{\mathrm{cr}}$ parameter rises in the thermal destruction phase due to the transformation of some small aromatic rings into large ones, a process that is influenced primarily by low-temperature CLRs (the rapid increase in cross-linking density facilitates a transformation from small aromatic rings to the relatively large ones). The growth in the number of crystalline carbons occurs parallel to these disruptive processes. The SO15 h samples contain more oxygenated functional groups (such as carboxyl) after pre-oxidation treatment. These oxygen functional groups, which may exist between layers or edge of layers in crystalline carbon, are broken to form large amounts of free radicals that participate in the development of cross-linkages between layers. Based on these factors, the mean size of the crystalline structures present increases during this stage.

The $C_{\mathrm{cr}}$ parameters vary little in the range from 350 to $500{ }^{\circ} \mathrm{C}$, while the $L_{\mathrm{c}}$ and $L_{\mathrm{a}}$ parameter decrease and the $d_{002}$ parameter increases to a maximum value near $500^{\circ} \mathrm{C}$. With these increases in heating temperature and time, the accompanying thermal decomposition processes are characterized by the breakage of cross-links and depolymerization of ordered structures, which results in united layers splitting into smaller units that change their orientations and positions to rearrange into microcrystalline structure without the growth of crystallites, while the loosening of microcrystalline structures leads to an increase in $d_{002}$.

The structural changes observed in the SO15 h samples are different from those of the S samples during the re-solidification phase. The $C_{\mathrm{cr}}$ and $I_{\mathrm{D}} / I_{\mathrm{G}}$ parameters decrease sharply, indicating the beginning of degradation processes in the crystalline carbon structure, while, large aromatic rings transform into some small ones within the crystallites. These changes may derive from the breakdown of cross-links within the basic crystallite structure units at high temperature. The $L_{\mathrm{c}}$ parameter decreases, as the fraction of carbon layers located at the edge of the crystalline structure do not produce coherent scattering during degradation processes. As a result, these layers do not take part in the formation of the reflex (002). 


\section{Conclusions}

1. The SO15 h sample, with O-containing functional groups, and the S sample, with more aliphatic $\mathrm{C}-\mathrm{H}$ groups, undergo low- and moderate-temperature cross-linking reactions during carbonization, respectively.

2. For the SO15 h samples, firstly, low-temperature cross-linking facilitates an increase in $C_{\mathrm{cr}}$ parameter and in the mean size of crystalline structures, precipitating the transformation of amorphous carbon into crystalline carbon. Then, the breakage of cross links and depolymerization results in layers splitting into smaller units that adjust their directions and positions without the growth of crystallites. Finally, these degradation processes lead to the transformation of crystalline structures into amorphous carbon at high temperature.

3. For the S samples, firstly, the breakage of weak bonds leads to the transformation of crystalline carbon into amorphous carbon during thermal decomposition. Then moderate-temperature cross-linking promotes the appearance of small layers in the amorphous carbon structure and the transformation of amorphous carbon into crystalline carbon between $400-550{ }^{\circ} \mathrm{C}$. Those small layers with disadvantageous positions or adverse orientations that form cross-linkages with their larger neighbors draw together rather than coalesce. Finally, as the carbonization temperature increases, the new and old crystallite nuclei rearranged orientations to coalesce and form larger layers, and the effect of the stretching of crystalline layers leads to a decrease in the number of layers.

\section{Acknowledgements}

This research is financially supported by the National Natural Science Foundation of China (51134015, 51276052 and 51376054). To whom correspondence should be addressed. E-mail: gaojh@hit.edu.cn

\section{References}

[1] Zhu Y.W, Gao J.H, Li Y, Sun F, Gao JM, Wu SH, Qin YK. Preparation of activated carbons for $\mathrm{SO}_{2}$ adsorption by $\mathrm{CO}_{2}$ and steam activation, J. Taiwan Inst. Chem. Engrs. 43 (2012) 112-119.

[2] Chattopadhyaya, G, Macdonald D.G, Bakhshi N.N, Mohammadzadeh J.S.S., Dalai A.K, Preparation and characterization of chars and activated carbons from Saskatchewan lignite, Fuel Process Technol,87(2006)997-1006.

[3] Wang M.J, Roberts D.G, Kochanek M.A, Harris D.J, Chang L.P, Li C.Z, Raman Spectroscopic Investigations into Links between Intrinsic Reactivity and Char Chemical Structure, Energ Fuel, 28 (2014)285-290.

[4]Wigmans T., Industrial aspects of production and use of activated carbons , Carbon, 27(1989)., 13-22

[5] Ndaji F.E, Thomas K.M., The effects of oxidation on the macromolecular structure of coal, Fuel, 74(1995)932-937.

[6]Lu L, Sahajawalla V, Kong C, Hawis D, Quantitative X-ray diffraction analysis and its application to various coals, Carbon, 39 (2001)1821-1833.

[7] Wang Z.Q, Bai Z.Q, Li W, Bai J, Guo Z.X, Chen H.K, Effects of ion-exchanged calcium, barium and magnesium on cross-linking reactions during direct liquefaction of oxidized lignite, Fuel Process Technol, 94(2012)34-39.

[8]Solomon P.R. Serio M.A, Despande G.V, Kroo E, Cross-linking reactions during coal conversion, Energ Fuel, 4(1990)42-54.

[9]Yürüm Y, Kramer R, Levy M, Interaction of kerogen and mineral matrix of an oil shale in 
oxidative atmosphere, Thermochim Acta, 94 (1985)285-293.

[10]Green P.D, Johnson C.A, Thomas K.M, Applications of laser Raman microprobe spectroscopy to the characterization of coals and cokes, Fuel, 62 (1983)1013-1023.

[11] Bar-ziv E, Zaida A, Salatino P, Senneca O, Diagnostics of carbon gasification by raman microprobe spectroscopy, P Combust Inst, 28(2000)2369-2374.

[12] Liotta R, Brons G, Isaacs J, Oxidative weathering of Illinois No. 6 coal, Fuel, 62 (1983781-791).

[13] Meng F.R, Yu J.L, Tahmasebi A, Han Y.N, Zhao H, Lucas J, Wall T, Characteristics of Chars from Low-Temperature Pyrolysis of Lignite, Energ Fuel, 28 (2014)275-284.

[14] de la Puente G., Marbàn G., Modelling of volatile product evolution in coal pyrolysis. The role of aerial oxidation, J Anal Appl Pyrol, 44 (1998)205-218.

[15] Joseph J.T, Forrai T.R, Effect of exchangeable cations on liquefaction of low rank coals, Fuel, 71 (1992)75-80.

[16] Rhoads C.A, Senftle J.T, Coleman M.M, Davis A, Painter P.C, Further studies of coal oxidation, Fuel, 62 (1983)387-1397.

[17] Zubkova V.V, Some aspects of structural transformations taking place in organic mass of Ukrainian coals during heating. Part 1. Study of structural transformations when heating coals of different caking capacity, Fuel, 84 (2005)741-754.

[18] Zerda T.W, John A, Chmura K, Raman studies of coals , Fuel, 60 (1981)375-378.

[19] Zhang Y.D, Kang X.J, Influence of Calcination and Acidification on Structural Characterization of Anyang Anthracites, Energ Fuel, 277(2013)191-7197. 